\title{
Port-Hamiltonian systems: an introductory survey
}

\author{
Arjan van der Schaft
}

\begin{abstract}
The theory of port-Hamiltonian systems provides a framework for the geometric description of network models of physical systems. It turns out that port-based network models of physical systems immediately lend themselves to a Hamiltonian description. While the usual geometric approach to Hamiltonian systems is based on the canonical symplectic structure of the phase space or on a Poisson structure that is obtained by (symmetry) reduction of the phase space, in the case of a port-Hamiltonian system the geometric structure derives from the interconnection of its sub-systems. This motivates to consider Dirac structures instead of Poisson structures, since this notion enables one to define Hamiltonian systems with algebraic constraints. As a result, any power-conserving interconnection of port-Hamiltonian systems again defines a port-Hamiltonian system.

The port-Hamiltonian description offers a systematic framework for analysis, control and simulation of complex physical systems, for lumped-parameter as well as for distributed-parameter models.
\end{abstract}

Mathematics Subject Classification (2000). Primary 93A30,70H05,70H45,70Q05, 70G45, 93B29, 37J60; Secondary 93C10, 93C15, 93C20, 37K05.

Keywords. Interconnection, Dirac structures, constrained systems, Hamiltonian DAEs, stabilization, boundary control, conservation laws.

\section{Introduction}

Historically, the Hamiltonian approach has its roots in analytical mechanics and starts from the principle of least action, and proceeds, via the Euler-Lagrange equations and the Legendre transform, towards the Hamiltonian equations of motion. On the other hand, the network approach stems from electrical engineering, and constitutes a cornerstone of mathematical systems theory. While most of the analysis of physical systems has been performed within the Lagrangian and Hamiltonian framework, the network point of view is prevailing in modelling and simulation of (complex) physical engineering systems.

The framework of port-Hamiltonian systems combines both points of view, by associating with the interconnection structure of the network model a geometric structure given by a (pseudo-) Poisson structure, or more generally a Dirac structure. The Hamiltonian dynamics is then defined with respect to this Dirac structure and the Hamiltonian given by the total stored energy. Furthermore, port-Hamiltonian systems are open dynamical systems, which interact with their environment through ports. Re-

Proceedings of the International Congress of Mathematicians, Madrid, Spain, 2006

(C) 2006 European Mathematical Society 
sistive effects are included by terminating some of these ports on energy-dissipating elements.

Dirac structures encompass the geometric structures which are classically being used in the geometrization of mechanics (that is, Poisson structures and pre-symplectic structures), and allow to describe the geometric structure of dynamical systems with algebraic constraints. Furthermore, Dirac structures allow to extend the Hamiltonian description of distributed-parameter systems to include variable boundary conditions, leading to distributed-parameter port-Hamiltonian systems with boundary ports.

Acknowledgements. This survey is based on joint work with several co-authors. In particular I thank Bernhard Maschke and Romeo Ortega for fruitful collaborations.

\section{Finite-dimensional port-Hamiltonian systems}

In this section we recapitulate the basics of finite-dimensional port-Hamiltonian systems. For more details we refer e.g. to [19], [17], [20], [33], [34], [30], [36], [12], [5].

2.1. From classical Hamiltonian equations to port-Hamiltonian systems. The standard Hamiltonian equations for a mechanical system are given as

$$
\begin{aligned}
& \dot{q}=\frac{\partial H}{\partial p}(q, p), \\
& \dot{p}=-\frac{\partial H}{\partial q}(q, p)+F
\end{aligned}
$$

where the Hamiltonian $H(q, p)$ is the total energy of the system, $q=\left(q_{1}, \ldots, q_{k}\right)^{T}$ are generalized configuration coordinates for the mechanical system with $k$ degrees of freedom, $p=\left(p_{1}, \ldots, p_{k}\right)^{T}$ is the vector of generalized momenta, and the input $F$ is the vector of external generalized forces. The state space of (1) with local coordinates $(q, p)$ is called the phase space.

One immediately derives the following energy balance:

$$
\frac{d}{d t} H=\frac{\partial^{T} H}{\partial q}(q, p) \dot{q}+\frac{\partial^{T} H}{\partial p}(q, p) \dot{p}=\frac{\partial^{T} H}{\partial p}(q, p) F=\dot{q}^{T} F
$$

expressing that the increase in energy of the system is equal to the supplied work (conservation of energy). This motivates to define the output of the system as $e=\dot{q}$ (the vector of generalized velocities). 
System (1) is more generally given in the following form

$$
\begin{aligned}
& \dot{q}=\frac{\partial H}{\partial p}(q, p), \quad(q, p)=\left(q_{1}, \ldots, q_{k}, p_{1}, \ldots, p_{k}\right), \\
& \dot{p}=-\frac{\partial H}{\partial q}(q, p)+B(q) f, \quad f \in \mathbb{R}^{m}, \\
& e=B^{T}(q) \frac{\partial H}{\partial p}(q, p) \quad\left(=B^{T}(q) \dot{q}\right), \quad e \in \mathbb{R}^{m},
\end{aligned}
$$

with $B(q) f$ denoting the generalized forces resulting from the input $f \in \mathbb{R}^{m}$. In case $m<k$ we speak of an underactuated system. Similarly to (2) we obtain the energy balance

$$
\frac{d H}{d t}(q(t), p(t))=e^{T}(t) f(t) .
$$

A further generalization is to consider systems which are described in local coordinates as

$$
\begin{array}{rlrl}
\dot{x} & =J(x) \frac{\partial H}{\partial x}(x)+g(x) f, & & x \in \mathcal{X}, f \in \mathbb{R}^{m}, \\
e & =g^{T}(x) \frac{\partial H}{\partial x}(x), & e \in \mathbb{R}^{m},
\end{array}
$$

where $J(x)$ is an $n \times n$ matrix with entries depending smoothly on $x$, which is assumed to be skew-symmetric, that is $J(x)=-J^{T}(x)$, and $x=\left(x_{1}, \ldots, x_{n}\right)$ are local coordinates for an $n$-dimensional state space manifold $\mathcal{X}$ (not necessarily evendimensional as above). Because of skew-symmetry of $J$ we easily recover the energybalance $\frac{d H}{d t}(x(t))=e^{T}(t) f(t)$. We call (5) a port-Hamiltonian system with structure matrix $J(x)$, input matrix $g(x)$, and Hamiltonian $H$ ([17], [19], [18]).

Remark 2.1. In many examples the structure matrix $J$ will additionally satisfy an integrability condition (the Jacobi-identity) allowing us to find by Darboux's theorem "canonical coordinates". In this case $J$ is the structure matrix of a Poisson structure on $X$.

Example 2.2. An important class of systems that naturally can be written as portHamiltonian systems, is constituted by mechanical systems with kinematic constraints [22]. Consider a mechanical system locally described by $k$ configuration variables $q=\left(q_{1}, \ldots, q_{k}\right)$. Suppose that there are constraints on the generalized velocities $\dot{q}$, described as

$$
A^{T}(q) \dot{q}=0,
$$

with $A(q)$ an $r \times k$ matrix of rank $r$ everywhere. The constraints (6) are called holonomic if it is possible to find new configuration coordinates $\bar{q}=\left(\bar{q}_{1}, \ldots, \bar{q}_{k}\right)$ such that the constraints are equivalently expressed as $\dot{\bar{q}}_{k-r+1}=\dot{\bar{q}}_{n-r+2}=\cdots=\dot{\bar{q}}_{k}=0$, in which case the kinematic constraints integrate to the geometric constraints

$$
\bar{q}_{k-r+1}=c_{k-r+1}, \ldots, \bar{q}_{k}=c_{k}
$$


for certain constants $c_{k-r+1}, \ldots, c_{k}$ determined by the initial conditions. Then the system reduces to an unconstrained system in the remaining configuration coordinates $\left(\bar{q}_{1}, \ldots, \bar{q}_{k-r}\right)$. If it is not possible to integrate the kinematic constraints as above, then the constraints are called nonholonomic. The equations of motion for the mechanical system with constraints (6) are given by the constrained Hamiltonian equations

$$
\begin{aligned}
& \dot{q}=\frac{\partial H}{\partial p}(q, p), \\
& \dot{p}=-\frac{\partial H}{\partial q}(q, p)+A(q) \lambda+B(q) f, \\
& e=B^{T}(q) \frac{\partial H}{\partial p}(q, p), \\
& 0=A^{T}(q) \frac{\partial H}{\partial p}(q, p) .
\end{aligned}
$$

The constrained state space is therefore given as the following subset of the phase space:

$$
\mathcal{X}_{c}=\left\{(q, p) \mid A^{T}(q) \frac{\partial H}{\partial p}(q, p)=0\right\} .
$$

One way of proceeding is to eliminate the constraint forces, and to reduce the equations of motion to the constrained state space, leading (see [32] for details) to a portHamiltonian system (5). The structure matrix of this reduced port-Hamiltonian system satisfies the Jacobi identity if and only if the constraints (6) are holonomic [32]. An alternative way of approaching the system (8) is to formalize it directly as an implicit port-Hamiltonian system (with respect to a Dirac structure), as will be the topic of Section 2.3.

2.2. From port-based network modelling to port-Hamiltonian systems. In this subsection we take a different point of view by emphasizing how port-Hamiltonian systems directly arise from port-based network models of physical systems.

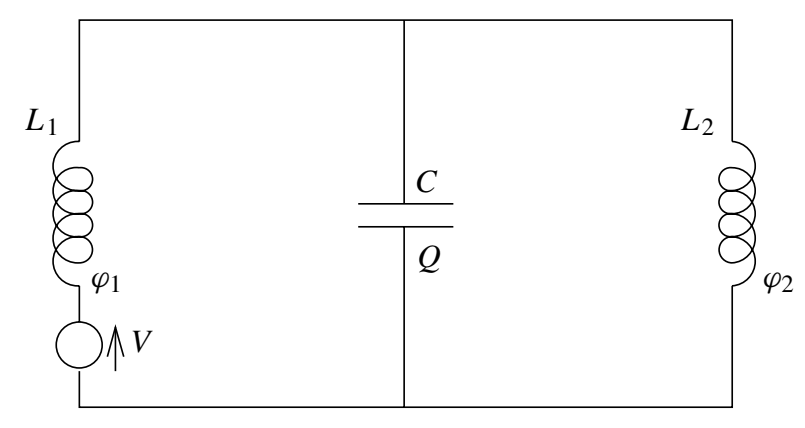

Figure 1. Controlled LC-circuit. 
In network models of complex physical systems the overall system is regarded as the interconnection of energy-storing elements via basic interconnection (balance) laws such as Newton's third law or Kirchhoff's laws, as well as power-conserving elements like transformers, kinematic pairs and ideal constraints, together with energydissipating elements [3], [14], [13]. The basic point of departure for the theory of port-Hamiltonian systems is to formalize the basic interconnection laws together with the power-conserving elements by a geometric structure, and to define the Hamiltonian as the total energy stored in the system. This is already illustrated by the following simple example.

Example 2.3 (LCTG circuits). Consider a controlled LC-circuit (see Figure 1) consisting of two inductors with magnetic energies $H_{1}\left(\varphi_{1}\right), H_{2}\left(\varphi_{2}\right)\left(\varphi_{1}\right.$ and $\varphi_{2}$ being the magnetic flux linkages), and a capacitor with electric energy $H_{3}(Q)$ ( $Q$ being the charge). If the elements are linear then $H_{1}\left(\varphi_{1}\right)=\frac{1}{2 L_{1}} \varphi_{1}^{2}, H_{2}\left(\varphi_{2}\right)=\frac{1}{2 L_{2}} \varphi_{2}^{2}$ and $H_{3}(Q)=\frac{1}{2 C} Q^{2}$. Furthermore let $V=u$ denote a voltage source. Using Kirchhoff's laws one obtains the dynamical equations

$$
\begin{aligned}
{\left[\begin{array}{c}
\dot{Q} \\
\dot{\varphi}_{1} \\
\dot{\varphi}_{2}
\end{array}\right] } & =\underbrace{\left[\begin{array}{ccc}
0 & 1 & -1 \\
-1 & 0 & 0 \\
1 & 0 & 0
\end{array}\right]}_{J}\left[\begin{array}{c}
\frac{\partial H}{\partial Q} \\
\frac{\partial H}{\partial \varphi_{1}} \\
\frac{\partial H}{\partial \varphi_{2}}
\end{array}\right]+\left[\begin{array}{l}
0 \\
1 \\
0
\end{array}\right] u, \\
y & =\frac{\partial H}{\partial \varphi_{1}} \quad \text { (= current through voltage source) }
\end{aligned}
$$

with $H\left(Q, \varphi_{1}, \varphi_{2}\right):=H_{1}\left(\varphi_{1}\right)+H_{2}\left(\varphi_{2}\right)+H_{3}(Q)$ the total energy. Clearly (by Tellegen's theorem) the matrix $J$ is skew-symmetric.

In this way every LC-circuit with independent elements can be modelled as a portHamiltonian system. Similarly any LCTG-circuit with independent elements can be modelled as a port-Hamiltonian system, with $J$ now being determined by Kirchhoff's laws and the constitutive relations of the transformers $T$ and gyrators $G$.

2.3. Dirac structures and implicit port-Hamiltonian systems. From a general modeling point of view physical systems are, at least in first instance, often described as DAE's, that is, a mixed set of differential and algebraic equations. This stems from the fact that in network modeling the system under consideration is regarded as obtained from interconnecting simpler sub-systems. These interconnections usually give rise to algebraic constraints between the state space variables of the sub-systems; thus leading to implicit systems. Therefore it is important to extend the framework of port-Hamiltonian systems to the context of implicit systems; that is, systems with algebraic constraints.

2.3.1. Dirac structures. In order to give the definition of an implicit port-Hamiltonian system we introduce the notion of a Dirac structure, formalizing the concept of 
a power-conserving interconnection, and generalizing the notion of a structure matrix $J(x)$ as encountered before.

Let $\mathcal{F}$ be an $\ell$-dimensional linear space, and denote its dual (the space of linear functions on $\mathcal{F}$ ) by $\mathcal{F}^{*}$. The product space $\mathcal{F} \times \mathcal{F}^{*}$ is considered to be the space of power variables, with power defined by

$$
P=\left\langle f^{*} \mid f\right\rangle, \quad\left(f, f^{*}\right) \in \mathcal{F} \times \mathcal{F}^{*},
$$

where $\left\langle f^{*} \mid f\right\rangle$ denotes the duality product. Often we call $\mathcal{F}$ the space of flows $f$, and $\mathcal{F}^{*}$ the space of efforts $e$, with the power of an element $(f, e) \in \mathcal{F} \times \mathcal{F}^{*}$ denoted as $\langle e \mid f\rangle$.

Example 2.4. Let $\mathcal{F}$ be the space of generalized velocities, and $\mathcal{F}^{*}$ be the space of generalized forces, then $\langle e \mid f\rangle$ is mechanical power. Similarly, let $\mathcal{F}$ be the space of currents, and $\mathcal{F}^{*}$ be the space of voltages, then $\langle e \mid f\rangle$ is electrical power.

There exists on $\mathcal{F} \times \mathcal{F}^{*}$ the canonically defined symmetric bilinear form

$$
\left\langle\left(f_{1}, e_{1}\right),\left(f_{2}, e_{2}\right)\right\rangle_{\mathcal{F} \times \mathcal{F} *}:=\left\langle e_{1} \mid f_{2}\right\rangle+\left\langle e_{2} \mid f_{1}\right\rangle
$$

for $f_{i} \in \mathcal{F}, e_{i} \in \mathcal{F}^{*}, i=1,2$.

Definition 2.5 ([6], [8], [7]). A constant Dirac structure on $\mathcal{F}$ is a linear subspace $\mathscr{D} \subset \mathcal{F} \times \mathcal{F}^{*}$ such that

$$
\mathscr{D}=\mathscr{D}^{\perp}
$$

where $^{\perp}$ denotes the orthogonal complement with respect to the bilinear form $\langle,\rangle_{\mathcal{F} \times \mathcal{F}^{*}}$.

It immediately follows that the dimension of any Dirac structure $\mathscr{D}$ on an $\ell$-dimensional linear space is equal to $\ell$. Furthermore, let $(f, e) \in \mathscr{D}=\mathscr{D}^{\perp}$. Then by (12)

$$
0=\langle(f, e),(f, e)\rangle_{\mathcal{F} \times \mathcal{F}^{*}}=2\langle e \mid f\rangle .
$$

Thus for all $(f, e) \in \mathscr{D}$ we obtain $\langle e \mid f\rangle=0$. Hence a Dirac structure $\mathscr{D}$ on $\mathcal{F}$ defines a power-conserving relation between the power variables $(f, e) \in \mathcal{F} \times \mathcal{F}^{*}$, which moreover has maximal dimension.

Remark 2.6. For many systems, especially those with 3-D mechanical components, the Dirac structure is actually modulated by the energy or geometric variables. Furthermore, the state space $\mathcal{X}$ is a manifold and the flows $f_{S}=-\dot{x}$ corresponding to energy-storage are elements of the tangent space $T_{x} \mathcal{X}$ at the state $x \in \mathcal{X}$, while the efforts $e_{S}$ are elements of the co-tangent space $T_{x}^{*} \mathcal{X}$.

Modulated Dirac structures often arise as a result of kinematic constraints. In many cases, these constraints will be configuration dependent, causing the Dirac structure to be modulated by the configuration variables, cf. Section 2.2. 
In general, a port-Hamiltonian system can be represented as in Figure 2. The port variables entering the Dirac structure $\mathscr{D}$ have been split in different parts. First, there are two internal ports. One, denoted by $\delta$, is corresponding to energy-storage and the other one, denoted by $\mathcal{R}$, is corresponding to internal energy-dissipation (resistive elements). Second, two external ports are distinguished. The external port denoted by $\mathcal{C}$ is the port that is accessible for controller action. Also the presence of sources may be included in this port. Finally, the external port denoted by $\mathcal{I}$ is the interaction port, defining the interaction of the system with (the rest of) its environment.

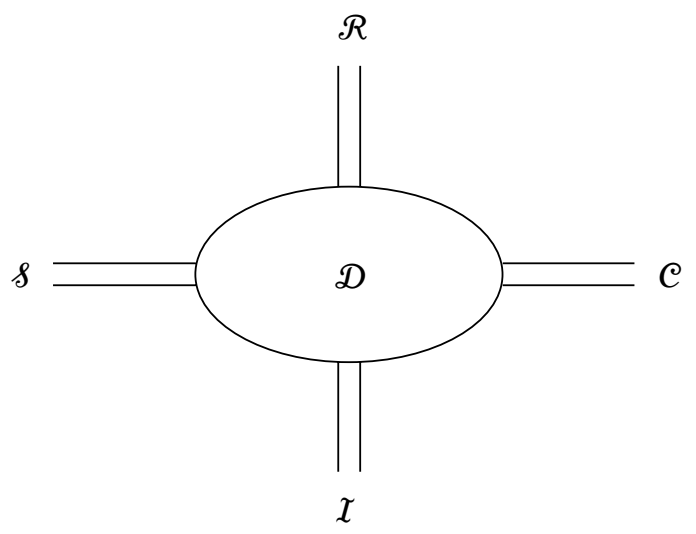

Figure 2. Port-Hamiltonian system.

2.3.2. Energy storage port. The port variables associated with the internal storage port will be denoted by $\left(f_{S}, e_{S}\right)$. They are interconnected to the energy storage of the system which is defined by a finite-dimensional state space manifold $\mathcal{X}$ with coordinates $x$, together with a Hamiltonian function $H: \mathcal{X} \rightarrow \mathbb{R}$ denoting the energy. The flow variables of the energy storage are given by the rate $\dot{x}$ of the energy variables $x$. Furthermore, the effort variables of the energy storage are given by the co-energy variables $\frac{\partial H}{\partial x}(x)$, resulting in the energy balance

$$
\frac{d}{d t} H=\left\langle\frac{\partial H}{\partial x}(x) \mid \dot{x}\right\rangle=\frac{\partial^{T} H}{\partial x}(x) \dot{x} .
$$

(Here we adopt the convention that $\frac{\partial H}{\partial x}(x)$ denotes the column vector of partial derivatives of $H$.)

The interconnection of the energy storing elements to the storage port of the Dirac structure is accomplished by setting

$$
\begin{aligned}
f_{S} & =-\dot{x}, \\
e_{S} & =\frac{\partial H}{\partial x}(x) .
\end{aligned}
$$


Hence the energy balance (15) can be also written as

$$
\frac{d}{d t} H=\frac{\partial^{T} H}{\partial x}(x) \dot{x}=-e_{S}^{T} f_{S} .
$$

2.3.3. Resistive port. The second internal port corresponds to internal energy dissipation (due to friction, resistance, etc.), and its port variables are denoted by $\left(f_{R}, e_{R}\right)$. These port variables are terminated on a static resistive relation $\mathcal{R}$. In general, a static resistive relation will be of the form

$$
R\left(f_{R}, e_{R}\right)=0,
$$

with the property that for all $\left(f_{R}, e_{R}\right)$ satisfying (18)

$$
\left\langle e_{R} \mid f_{R}\right\rangle \leq 0 \text {. }
$$

In many cases we may restrict ourselves to linear resistive relations. This means that the resistive port variables $\left(f_{R}, e_{R}\right)$ satisfy linear relations of the form

$$
R_{f} f_{R}+R_{e} e_{R}=0
$$

The inequality (19) corresponds to the square matrices $R_{f}$ and $R_{e}$ satisfying the properties of symmetry and semi-positive definiteness

$$
R_{f} R_{e}^{T}=R_{e} R_{f}^{T} \geq 0,
$$

together with the dimensionality condition $\operatorname{rank}\left[R_{f} \mid R_{e}\right]=\operatorname{dim} f_{R}$.

Without the presence of additional external ports, the Dirac structure of the portHamiltonian system satisfies the power-balance $e_{S}^{T} f_{S}+e_{R}^{T} f_{R}=0$ which leads to

$$
\frac{d}{d t} H=-e_{S}^{T} f_{S}=e_{R}^{T} f_{R} \leq 0 .
$$

An important special case of resistive relations between $f_{R}$ and $e_{R}$ occurs when the resistive relations can be expressed as an input-output mapping $f_{R}=-F\left(e_{R}\right)$, where the resistive characteristic $F: \mathbb{R}^{m_{r}} \rightarrow \mathbb{R}^{m_{r}}$ satisfies

$$
e_{R}^{T} F\left(e_{R}\right) \geq 0, \quad e_{R} \in \mathbb{R}^{m_{r}}
$$

For linear resistive elements this specializes to $f_{R}=-\tilde{R} e_{R}$, for some positive semidefinite symmetric matric $\tilde{R}=\tilde{R}^{T} \geq 0$.

2.3.4. External ports. Now, let us consider in more detail the external ports to the system. We distinguish between two types of external ports. One is the control port $\mathcal{C}$, with port variables $\left(f_{C}, e_{C}\right)$, which are the port variables which are accessible for controller action. Other type of external port is the interaction port $\mathcal{L}$, which denotes the interaction of the port-Hamiltonian system with its environment. The 
port variables corresponding to the interaction port are denoted by $\left(f_{I}, e_{I}\right)$. By taking both the external ports into account the power-balance extends to

$$
e_{S}^{T} f_{S}+e_{R}^{T} f_{R}+e_{C}^{T} f_{C}+e_{I}^{T} f_{I}=0
$$

whereby (22) extends to

$$
\frac{d}{d t} H=e_{R}^{T} f_{R}+e_{C}^{T} f_{C}+e_{I}^{T} f_{I}
$$

2.3.5. Port-Hamiltonian dynamics. The port-Hamiltonian system with state space $\mathcal{X}$, Hamiltonian $H$ corresponding to the energy storage port $\&$, resistive port $\mathcal{R}$, control port $\mathcal{C}$, interconnection port $\mathcal{L}$, and total Dirac structure $\mathscr{D}$ will be succinctly denoted by $\Sigma=(\mathcal{X}, H, \mathcal{R}, \mathcal{C}, \mathcal{I}, \mathbb{D})$. The dynamics of the port-Hamiltonian system is specified by considering the constraints on the various port variables imposed by the Dirac structure, that is

$$
\left(f_{S}, e_{S}, f_{R}, e_{R}, f_{C}, e_{C}, f_{I}, e_{I}\right) \in \mathscr{D},
$$

and to substitute in these relations the equalities $f_{S}=-\dot{x}, e_{S}=\frac{\partial H}{\partial x}(x)$. This leads to the implicitly defined dynamics

$$
\left(-\dot{x}(t), \frac{\partial H}{\partial x}(x(t)), f_{R}(t), e_{R}(t), f_{C},(t), e_{C}(t), f_{I}(t), e_{I}(t)\right) \in \mathscr{D}
$$

with $f_{R}(t), e_{R}(t)$ satisfying for all $t$ the resistive relation (18):

$$
R\left(f_{R}, e_{R}\right)=0 .
$$

In many cases of interest the dynamics (26) will constrain the allowed states $x$, depending on the values of the external port variables $\left(f_{C}, e_{C}\right)$ and $\left(f_{I}, e_{I}\right)$. Thus in an equational representation port-Hamiltonian systems generally will consist of a mixed set of differential and algebraic equations (DAEs).

Example 2.7 (General LC- circuits). Consider an LC-circuit with general network topology. Kirchhoff's current and voltage laws take the general form

$$
\begin{gathered}
A_{L}{ }^{T} I_{L}+A_{C}^{T} I_{C}+A_{P}{ }^{T} I_{P}=0, \\
V_{L}=A_{L} \lambda, \quad V_{C}=A_{C} \lambda, \quad V_{P}=A_{P} \lambda
\end{gathered}
$$

for some matrices $A_{L}, A_{C}, A_{S}$. Here $I_{L}, I_{C}, I_{P}$ denote the currents, respectively through the inductors, capacitors and external ports. Likewise, $V_{L}, V_{C}, V_{P}$ denote the voltages over the inductors, capacitors and external ports. Kirchhoff's current and voltage laws define a Dirac structure between the flows and efforts:

$$
\begin{aligned}
f & =\left(I_{C}, V_{L}, I_{P}\right)=\left(-\dot{Q},-\dot{\phi}, I_{P}\right), \\
e & =\left(V_{C}, I_{L}, V_{P}\right)=\left(\frac{\partial H}{\partial Q}, \frac{\partial H}{\partial \phi}, V_{P}\right)
\end{aligned}
$$


with Hamiltonian $H(\phi, Q)$ the total energy. This leads the to port-Hamiltonian system in implicit form

$$
\begin{aligned}
-\dot{\phi} & =A_{L} \lambda, \\
\frac{\partial H}{\partial Q} & =A_{C} \lambda, \\
V_{P} & =A_{P} \lambda, \\
0 & =A_{L}{ }^{T} \frac{\partial H}{\partial \phi}-A_{C}^{T} \dot{Q}+A_{P}^{T} I_{P} .
\end{aligned}
$$

Example 2.8 (Electro-mechanical system). Consider the dynamics of an iron ball in the magnetic field of a controlled inductor: The port-Hamiltonian description of this

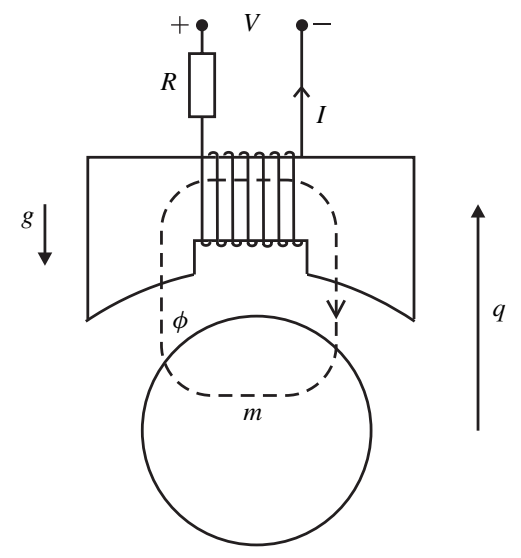

Figure 3. Magnetically levitated ball.

system (with $q$ the height of the ball, $p$ the vertical momentum, and $\varphi$ the magnetic flux of the inductor) is given as

$$
\begin{aligned}
{\left[\begin{array}{c}
\dot{q} \\
\dot{p} \\
\dot{\varphi}
\end{array}\right] } & =\left[\begin{array}{ccc}
0 & 1 & 0 \\
-1 & 0 & 0 \\
0 & 0 & -\frac{1}{R}
\end{array}\right]\left[\begin{array}{c}
\frac{\partial H}{\partial q} \\
\frac{\partial H}{\partial p} \\
\frac{\partial H}{\partial \varphi}
\end{array}\right]+\left[\begin{array}{l}
0 \\
0 \\
1
\end{array}\right] V, \\
I & =\frac{\partial H}{\partial \varphi} .
\end{aligned}
$$

This is a typical example of a system where the coupling between two different physical domains (mechanical and magnetic) takes place via the Hamiltonian

$$
H(q, p, \varphi)=m g q+\frac{p^{2}}{2 m}+\frac{\varphi^{2}}{2 k_{1}\left(1-\frac{q}{k_{2}}\right)}
$$


where the last term depends both on a magnetic variable (in this case $\varphi$ ) and a mechanical variable (in this case the height $q$ ).

2.4. Input-state-output port-Hamiltonian systems. An important special case of port-Hamiltonian systems is the class of input-state-output port-Hamiltonian systems, where there are no algebraic constraints on the state space variables, and the flow and effort variables of the resistive, control and interaction port are split into conjugated input-output pairs. Input-state-output port-Hamiltonian systems without interaction port are of the form

$$
\begin{aligned}
& \dot{x}=[J(x)-R(x)] \frac{\partial H}{\partial x}(x)+g(x) u, \\
& y=g^{T}(x) \frac{\partial H}{\partial x}(x)
\end{aligned}
$$

where $u, y$ are the input-output pairs corresponding to the control port $\mathcal{C}$. Here the matrix $J(x)$ is skew-symmetric, while the matrix $R(x)=R^{T}(x) \geq 0$ specifies the resistive structure, and is given as $R(x)=g_{R}^{T}(x) \tilde{R} g_{R}(x)$ for some linear resistive relation $f_{R}=-\tilde{R} e_{R}, \tilde{R}=\tilde{R}^{T} \geq 0$, with $g_{R}$ representing the input matrix corresponding to the resistive port. The underlying Dirac structure of the system is then given by the graph of the skew-symmetric linear map

$$
\left(\begin{array}{ccc}
-J(x) & -g_{R}(x) & -g(x) \\
g_{R}^{T}(x) & 0 & 0 \\
g^{T}(x) & 0 & 0
\end{array}\right) .
$$

\section{Control by interconnection of port-Hamiltonian systems}

The basic property of port-Hamiltonian systems is that the power-conserving interconnection of any number of port-Hamiltonian systems is again a port-Hamiltonian system.

To be explicit, consider two port-Hamiltonian systems $\Sigma_{A}$ and $\Sigma_{B}$ with Dirac structures $\mathscr{D}_{A}$ and $\mathscr{D}_{B}$ and Hamiltonians $H_{A}$ and $H_{B}$, defined on state spaces $\mathcal{X}_{A}$, respectively $\mathcal{X}_{B}$. For convenience, split the ports of the Dirac structures $\mathscr{D}_{A}$ and $\mathscr{D}_{B}$ into the internal energy storage ports and all remaining external ports whose port-variables are denoted respectively by $f_{A}, e_{A}$ and $f_{B}, e_{B}$. Now, consider any interconnection Dirac structure $\mathscr{D}_{I}$ involving the port-variables $f_{A}, e_{A}, f_{B}, e_{B}$ possibly together with additional port-variables $f_{I}, e_{I}$. Then the interconnection of the systems $\Sigma_{A}$ and $\Sigma_{B}$ via $\mathscr{D}_{I}$ is again a port-Hamiltonian system with respect to the composed Dirac structure $\mathscr{D}_{A} \circ \mathscr{D}_{I} \circ \mathscr{D}_{B}$, involving as port-variables the internal storage port-variables of $\mathscr{D}_{A}$ and $\mathscr{D}_{B}$ together with the additional port-variables $F_{I}, e_{I}$. For details we refer to [5], [34], [30]. 
Furthermore, the state space of the interconnected port-Hamiltonian system is the product of the two state spaces $\mathcal{X}_{A} \times \mathcal{X}_{B}$, while its Hamiltonian is simply the sum $H_{A}+H_{B}$ of the two Hamiltonians.

This basic statement naturally extends to the interconnection of any number of port-Hamiltonian systems via an interconnection Dirac structure.

Control by port-interconnection is based on designing a controller system which is interconnected to the control port with port-variables $\left(f_{C}, e_{C}\right)$. In principle this implies that we only consider collocated control, where the controller will only use the information about the plant port-Hamiltonian system that is contained in the conjugated pairs $\left(f_{C}, e_{C}\right)$ of port variables of the control port, without using additional information about the plant (e.g. corresponding to observation on other parts of the plant system). In the second place, we will restrict attention to controller systems which are themselves also port-Hamiltonian systems. There are two main reasons for this. One is that by doing so the closed-loop system is again a port-Hamiltonian system, allowing to easily ensure some desired properties. Furthermore, it will turn out that the port-Hamiltonian framework suggests useful ways to construct port-Hamiltonian controller systems. Second reason is that port-Hamiltonian controller systems allow in principle for a physical system realization (thus linking to passive control and systems design) and physical interpretation of the controller action.

Since we do not know the environment (or only have very limited information about it), but on the other hand, the system will interact with this unknown environment, the task of the controller is often two-fold: 1) to achieve a desired control goal (e.g. setpoint regulation or tracking) if the interaction with the environment is marginal or can be compensated, 2) to make sure that the controlled system has a desired interaction behavior with its environment. It is fair to say that up to now the development of the theory of control of port-Hamiltonian systems has mostly concentrated on the second aspect (which at the same time, is often underdeveloped in other control theories).

Most successful approaches to deal with the second aspect of the control goal are those based on the concept of "passivity", such as dissipativity theory [38], impedance control [13] and Intrinsically Passive Control (IPC) [36]. In fact, the port-Hamiltonian control theory can be regarded as an enhancement to the theory of passivity, making a much closer link with complex physical systems modeling at one hand and with the theory of dynamical systems (in particular, Hamiltonian dynamics) at the other hand.

As said above, we will throughout consider controller systems which are again port-Hamiltonian systems. We will use the same symbols as above for the internal and external ports and port-variables of the controller port-Hamiltonian system, with an added overbar ${ }^{-}$or a superscript ${ }^{c}$ in order to distinguish it from the plant system. (The interaction port of the controller system may be thought of as an extra possibility for additional controller action (outer-loop control).) In order to further distinguish the plant system and the controller we denote the state space of the plant system by $\mathcal{X}_{p}$ with coordinates $x_{p}$, the Dirac structure by $\mathscr{D}_{p}$ and its Hamiltonian by $H_{p}$, while we will denote the state space manifold of the controller system by $X_{c}$ with coordinates $x_{c}$, its Dirac structure by $\mathscr{D}_{c}$ and its Hamiltonian by $H_{c}: X_{c} \rightarrow \mathbb{R}$. The interconnection 
of the plant port-Hamiltonian system with the controller port-Hamiltonian system is obtained by equalizing the port variables at the control port by

$$
\begin{aligned}
& f_{C}=-\bar{f}_{C}, \\
& e_{C}=\bar{e}_{C}
\end{aligned}
$$

where $\bar{f}_{C}, \bar{e}_{C}$ denote the control port variables of the controller system. Here, the minus sign is inserted to have a uniform notion of direction of power flow. Clearly, this 'synchronizing' interconnection is power-conserving, that is $e_{C}^{T} f_{C}+\bar{e}_{C}^{T} \bar{f}_{C}=0$.

Remark 3.1. A sometimes useful alternative is the gyrating power-conserving interconnection

$$
\begin{aligned}
& f_{C}=-\bar{e}_{C}, \\
& e_{C}=\bar{f}_{C} .
\end{aligned}
$$

In fact, the standard feedback interconnection can be regarded to be of this type.

For both interconnection constraints it directly follows from the theory of composition of Dirac structures that the interconnected (closed-loop) system is again a port-Hamiltonian system with Dirac structure determined by the Dirac structures of the plant PH system and the controller PH system.

The resulting interconnected $\mathrm{PH}$ system has state space $\mathcal{X}_{p} \times \mathcal{X}_{c}$, Hamiltonian $H_{p}+H_{c}$, resistive ports $\left(f_{R}, e_{R}, \bar{f}_{R}, \bar{e}_{R}\right)$ and interaction ports $\left(f_{I}, e_{I}, \bar{f}_{I}, \bar{e}_{I}\right)$, satisfying the power-balance

$$
\frac{d}{d t}\left(H_{p}+H_{c}\right)=e_{R}^{T} f_{R}+\bar{e}_{R}^{T} \bar{f}_{R}+e_{I}^{T} f_{I}+\bar{e}_{I}^{T} \bar{f}_{I} \leq e_{I}^{T} f_{I}+\bar{e}_{I}^{T} \bar{f}_{I}
$$

since both $e_{R}^{T} f_{R} \leq 0$ and $\bar{e}_{R}^{T} \bar{f}_{R} \leq 0$. Hence we immediately recover the state space formulation of the passivity theorem, see e.g. [31], if $H_{p}$ and $H_{c}$ are both nonnegative, implying that the plant and the controller system are passive (with respect to their controller and interaction ports and storage functions $H_{p}$ and $H_{c}$ ), then also the closed -loop system is passive (with respect to the interaction ports and storage function $H_{p}+H_{c}$.)

Furthermore, due to the Hamiltonian structure, we can go beyond the passivity theorem, and we can derive conditions which ensure that we can passify and/or stabilize plant port-Hamiltonian systems for which the Hamiltonian $H_{p}$ does not have a minimum at the desired equilibrium.

3.1. Stabilization by Casimir generation. What does the power-balance (33)mean for the stability properties of the closed-loop system, and how can we design the controller port-Hamiltonian system in such a way that the closed-loop system has desired stability properties? Let us first consider the stability of an arbitrary portHamiltonian system $\Sigma=(\mathcal{X}, H, \mathcal{R}, \mathcal{C}, \mathcal{I}, \mathscr{D})$ without control or interaction ports, 
that is, an autonomous port-Hamiltonian system $\Sigma=(\mathcal{X}, H, \mathcal{R}, \mathscr{D})$. Clearly, the power-balance (33) reduces to

$$
\frac{d}{d t} H=e_{R}^{T} f_{R} \leq 0
$$

Hence we immediately infer by standard Lyapunov theory that if $x^{*}$ is a minimum of the Hamiltonian $H$ then it will be a stable equilibrium of the autonomous portHamiltonian system $\Sigma=(\mathcal{X}, H, \mathcal{R}, \mathcal{D})$, which is actually asymptotically stable if the dissipation term $e_{R}^{T} f_{R}$ is negative definite outside $x^{*}$, or alternatively if some sort of detectability condition is satisfied, guaranteeing asymptotic stability by the use of LaSalle's Invariance principle (see for details e.g. [31]).

However, what can we say if $x^{*}$ is not a minimum of $H$, and thus we cannot directly use $H$ as a Lyapunov function?

A well-known method in Hamiltonian systems, sometimes called the EnergyCasimir method, is to use in the Lyapunov analysis next to the Hamiltonian other conserved quantities (dynamical invariants) which may be present in the system. Indeed, if we may find other conserved quantities then candidate Lyapunov functions can be sought within the class of combinations of the Hamiltonian $H$ and those conserved quantities. In particular, if we can find a conserved quantity $C: \mathcal{X} \rightarrow \mathbb{R}$ such that $V:=H+C$ has a minimum at the desired equilibrium $x^{*}$ then we can still infer stability or asymptotic stability by replacing (34) by

$$
\frac{d}{d t} V=e_{R}^{T} f_{R} \leq 0
$$

and thus using $V$ as a Lyapunov function.

For the application of the Energy-Casimir method one may distinguish between two main cases. First situation occurs if the desired equilibrium $x^{*}$ is not a stationary point of $H$, and one looks for a conserved quantity $C$ such that $H+C$ has a minimum at $x^{*}$. This for example happens in the case that the desired set-point $x^{*}$ is not an equilibrium of the uncontrolled system, but only a controlled equilibrium of the system. Second situation occurs when $x^{*}$ is a stationary point of $H$, but not a minimum.

Functions that are conserved quantities of the system for every Hamiltonian are called Casimir functions or simply Casimirs. Casimirs are completely characterized by the Dirac structure of the port-Hamiltonian system. Indeed, a function $C: \mathcal{X} \rightarrow \mathbb{R}$ is a Casimir function of the autonomous port-Hamiltonian system (without energy dissipation) $\Sigma=(\mathcal{X}, H, \mathscr{D})$ if and only if the gradient vector $e=\frac{\partial^{T} C}{\partial x}$ satisfies

$$
e^{T} f_{S}=0 \text { for all } f_{S} \text { for which there exists } e_{S} \text { such that }\left(f_{S}, e_{S}\right) \in \mathscr{D} \text {. }
$$

Indeed, (36) is equivalent to

$$
\frac{d}{d t} C=\frac{\partial^{T} C}{\partial x}(x(t)) \dot{x}(t)=\frac{\partial^{T} C}{\partial x}(x(t)) f_{S}=e^{T} f_{S}=0
$$


for every port-Hamiltonian system $(\mathcal{X}, H, \mathscr{D})$ with the same Dirac structure $\mathscr{D}$. By the generalized skew-symmetry of the Dirac structure (36) is equivalent to the requirement that $e=\frac{\partial^{T} C}{\partial x}$ satisfies

$$
(0, e) \in \mathscr{D} \text {. }
$$

Similarly, we define a Casimir function for a port-Hamiltonian system with dissipation $\Sigma=(\mathcal{X}, H, \mathcal{R}, \mathcal{D})$ to be any function $C: \mathcal{X} \rightarrow \mathbb{R}$ satisfying

$$
(0, e, 0,0) \in \mathcal{D} \text {. }
$$

Indeed, this will imply that

$$
\frac{d}{d t} C=\frac{\partial^{T} C}{\partial x}(x(t)) \dot{x}(t)=\frac{\partial^{T} C}{\partial x}(x(t)) f_{p}=e^{T} f_{p}=0
$$

for every port-Hamiltonian system $(\mathcal{X}, H, \mathcal{R}, \mathcal{D})$ with the same Dirac structure $\mathscr{D}$. (In fact by definiteness of the resistive structures the satisfaction of (39) for a particular resistive structure $\mathcal{R}$ implies the satisfaction for all resistive structures $\mathcal{R}$.)

Now let us come back to the design of a controller port-Hamiltonian system such that the closed-loop system has desired stability properties. Suppose we want to stabilize the plant port-Hamiltonian system $\left(X_{p}, H_{p}, \mathcal{R}, \mathcal{C}, \mathscr{D}_{p}\right)$ around a desired equilibrium $x_{p}^{*}$. We know that for every controller port-Hamiltonian system the closed-loop system satisfies

$$
\frac{d}{d t}\left(H_{p}+H_{c}\right)=e_{R}^{T} f_{R}+\bar{e}_{R}^{T} \bar{f}_{R} \leq 0
$$

What if $x^{*}$ is not a minimum for $H_{p}$ ? A possible strategy is to generate Casimir functions $C\left(x_{p}, x_{c}\right)$ for the closed-loop system by choosing the controller port-Hamiltonian system in an appropriate way. Thereby we generate candidate Lyapunov functions for the closed-loop system of the form

$$
V\left(x_{p}, x_{c}\right):=H_{p}\left(x_{p}\right)+H_{c}\left(x_{c}\right)+C\left(x_{p}, x_{c}\right)
$$

where the controller Hamiltonian function $H_{c}: X_{c} \rightarrow \mathbb{R}$ still has to be designed. The goal is thus to construct a function $V$ as above in such a way that $V$ has a minimum at $\left(x_{p}^{*}, x_{c}^{*}\right)$ where $x_{c}^{*}$ still remains to be chosen. This strategy thus is based on finding all the achievable closed-loop Casimirs. Furthermore, since the closed-loop Casimirs are based on the closed-loop Dirac structures, this reduces to finding all the achievable closed-loop Dirac structures $\mathcal{D} \circ \overline{\mathcal{D}}$.

Another way to interpret the generation of Casimirs for the closed-loop system is to look at the level sets of the Casimirs as invariant submanifolds of the combined plant and controller state space $\mathcal{X}_{p} \times \mathcal{X}_{c}$. Restricted to every such invariant submanifold (part of) the controller state can be expressed as a function of the plant state, whence the closed-loop Hamiltonian restricted to such an invariant manifold can be seen as a 
shaped version of the plant Hamiltonian. To be explicit (see e.g. [31], [24], [25] for details) suppose that we have found Casimirs of the form

$$
x_{c i}-F_{i}\left(x_{p}\right), \quad i=1, \ldots, n_{p}
$$

where $n_{p}$ is the dimension of the controller state space, then on every invariant manifold $x_{c i}-F_{i}\left(x_{p}\right)=\alpha_{i}, i=1, \ldots, n_{p}$, where $\alpha=\left(\alpha_{1}, \ldots, \alpha_{n_{p}}\right)$ is a vector of constants depending on the initial plant and controller state, the closed-loop Hamiltonian can be written as

$$
H_{s}\left(x_{p}\right):=H_{p}\left(x_{p}\right)+H_{c}\left(F\left(x_{p}\right)+\alpha\right),
$$

where, as before, the controller Hamiltonian $H_{c}$ still can be assigned. This can be regarded as shaping the original plant Hamiltonian $H_{p}$ to a new Hamiltonian $H_{s}$.

3.2. Port Control. In broad terms, the Port Control problem is to design, given the plant port-Hamiltonian system, a controller port-Hamiltonian system such that the behavior at the interaction port of the plant port-Hamiltonian system is a desired one, or close to a desired one. This means that by adding the controller system we seek to shape the external behavior at the interaction port of the plant system. If the desired external behavior at this interaction port is given in input-output form as a desired (dynamic) impedance, then this amounts to the Impedance Control problem as introduced and studied by Hogan and co-workers [13]; see also [36] for subsequent developments.

The Port Control problem, as stated in this generality, immediately leads to two fundamental questions: 1). Given the plant PH system, and the controller PH system to be arbitrarily designed, what are the achievable behaviors of the closed-loop system at the interaction port of the plant? 2). If the desired behavior at the interaction port of the plant is not achievable, then what is the closest achievable behavior? Of course, the second question leaves much room for interpretation, since there is no obvious interpretation of what we mean by 'closest behavior'. Also the first question in its full generality is not easy to answer, and we shall only address an important subproblem.

An obvious observation is that the desired behavior, in order to be achievable, needs to be the port behavior of a PH system. This leads already to the problem of characterizing those external behaviors which are port behaviors of port-Hamiltonian systems. Secondly, the Port Control problem can be split into a number of subproblems. Indeed, we know that the closed-loop system arising from interconnection of the plant PH system with the controller PH system is specified by a Hamiltonian which is just the sum of the plant Hamiltonian and the controller Hamiltonian, and a resistive structure which is the "product" of the resistive structure of the plant and of the controller system, together with a Dirac structure which is the composition of the plant Dirac structure and the controller Dirac structure. Therefore an important subproblem is again to characterize the achievable closed-loop Dirac structures. On the other hand, a fundamental problem in addressing the Port Control problem in general theoretical terms is the lack of a systematic way to specify 'desired behavior'. 
The problem of Port Control is to determine the controller system in such a way that the port behavior in the port variables $f_{I}, e_{I}$ is a desired one. In this particular (simple and linear) example the desired behavior can be quantified e.g. in terms of a desired stiffness and damping of the closed-loop system, which is easily expressed in terms of the closed-loop transfer function from $f_{I}$ to $e_{I}$. Of course, on top of the requirements on the closed-loop transfer function we would also require internal stability of the closed-loop system. For an appealing example of port control of port-Hamiltonian systems within a context of hydraulic systems we refer to [15].

3.3. Energy Control. Consider two port-Hamiltonian systems $\Sigma_{i}$ (without internal dissipation) in input-state-output form

$$
\begin{aligned}
& \dot{x}_{i}=J_{i}\left(x_{i}\right) \frac{\partial H_{i}}{\partial x_{i}}+g_{i}\left(x_{i}\right) u_{i}, \\
& y_{i}=g_{i}^{T}\left(x_{i}\right) \frac{\partial H_{i}}{\partial x_{i}}, \quad i=1,2,
\end{aligned}
$$

both satisfying the power-balance $\frac{d}{d t} H_{i}=y_{i}^{T} u_{i}$. Suppose now that we want to transfer the energy from the port-Hamiltonian system $\Sigma_{1}$ to the port-Hamiltonian system $\Sigma_{2}$, while keeping the total energy $H_{1}+H_{2}$ constant. This can be done by using the following output feedback

$$
\left[\begin{array}{l}
u_{1} \\
u_{2}
\end{array}\right]=\left[\begin{array}{cc}
0 & -y_{1} y_{2}^{T} \\
y_{2} y_{1}^{T} & 0
\end{array}\right]\left[\begin{array}{l}
y_{1} \\
y_{2}
\end{array}\right] .
$$

Since the matrix in (42) is skew-symmetric it immediately follows that the closed-loop system composed of systems $\Sigma_{1}$ and $\Sigma_{2}$ linked by the power-conserving feedback is energy-preserving, that is $\frac{d}{d t}\left(H_{1}+H_{2}\right)=0$. However, if we consider the individual energies then we notice that

$$
\frac{d}{d t} H_{1}=-y_{1}^{T} y_{1} y_{2}^{T} y_{2}=-\left\|y_{1}\right\|^{2}\left\|y_{2}\right\|^{2} \leq 0
$$

implying that $H_{1}$ is decreasing as long as $\left\|y_{1}\right\|$ and $\left\|y_{2}\right\|$ are different from 0 . Conversely, as expected since the total energy is constant,

$$
\frac{d}{d t} H_{2}=y_{2}^{T} y_{2} y_{1}^{T} y_{1}=\left\|y_{2}\right\|^{2}\left\|y_{1}\right\|^{2} \geq 0
$$

implying that $H_{2}$ is increasing at the same rate. In particular, if $H_{1}$ has a minimum at the zero equilibrium, and $\Sigma_{1}$ is zero-state observable, then all the energy $H_{1}$ of $\Sigma_{1}$ will be transferred to $\Sigma_{2}$, provided that $\left\|y_{2}\right\|$ is not identically zero (which again can be guaranteed by assuming that $H_{2}$ has a minimum at the zero equilibrium, and that $\Sigma_{2}$ is zero-state observable).

If there is internal energy dissipation, then this energy transfer mechanism still works. However, the fact that $H_{2}$ grows or not will depend on the balance between the energy delivered by $\Sigma_{1}$ to $\Sigma_{2}$ and the internal loss of energy in $\Sigma_{2}$ due to dissipation. 
We conclude that this particular scheme of power-conserving energy transfer is accomplished by a skew-symmetric output feedback, which is modulated by the values of the output vectors of both systems. Of course this raises, among others, the question of the efficiency of the proposed energy-transfer scheme, and the need for a systematic quest of similar power-conserving energy-transfer schemes. We refer to [9] for a similar but different energy-transfer scheme directly motivated by the structure of the example (control of a snakeboard).

3.4. Achievable closed-loop Dirac structures. In all the control problems discussed above the basic question comes up what are the achievable closed-loop Dirac structures based on a given plant Dirac structure and a controller Dirac structure, which still is to be determined.

Theorem 3.2 ([5]). Given any plant Dirac structure $\mathscr{D}_{p}$, a certain interconnected $\mathscr{D}=\mathscr{D}_{p} \circ \mathscr{D}_{c}$ can be achieved by a proper choice of the controller Dirac structure $\mathscr{D}_{c}$ if and only if the following two equivalent conditions are satisfied:

$$
\begin{aligned}
& \mathscr{D}_{p}^{0} \subset \mathscr{D}^{0}, \\
& D^{\pi} \subset \mathscr{D}_{p}^{\pi}
\end{aligned}
$$

where

$$
\begin{aligned}
& \left.\mathscr{D}_{p}^{0}:=\left\{f_{1}, e_{1}\right) \mid\left(f_{1}, e_{1}, 0,0\right) \in \mathcal{D}_{p}\right\}, \\
& \mathscr{D}_{p}^{\pi}:=\left\{\left(f_{1}, e_{1}\right) \mid \text { there exists }\left(f_{2}^{P}, e_{2}^{P}\right) \text { with }\left(f_{1}, e_{1}, f_{2}^{P}, e_{2}^{P}\right) \in \mathcal{D}_{p}\right\}, \\
& \mathscr{D}^{0}:=\left\{\left(f_{1}, e_{1}\right) \mid\left(f_{1}, e_{1}, 0,0\right) \in D\right\}, \\
& \mathcal{D}^{\pi}:=\left\{\left(f_{1}, e_{1}\right) \mid \text { there exists }\left(f_{3}, e_{3}\right) \text { with }\left(f_{1}, e_{1}, f_{3}, e_{3}\right) \in \mathscr{D}\right\} .
\end{aligned}
$$

An important application of the above theorem concerns the characterization of Casimir functions which can be achieved by interconnecting a given plant portHamiltonian system with a controller port-Hamiltonian system.

\section{Distributed-parameter port-Hamiltonian systems}

The treatment of infinite-dimensional Hamiltonian systems in the literature is mostly confined to systems with boundary conditions such that the energy exchange through the boundary is zero. On the other hand, in many applications the interaction with the environment (e.g. actuation or measurement) will actually take place through the boundary of the system. In [35] a framework has been developed to represent classes of physical distributed-parameter systems with boundary energy flow as infinite-dimensional port-Hamiltonian systems. It turns out that in order to allow the inclusion of boundary variables in distributed-parameter systems the concept of (an 
infinite-dimensional) Dirac structure provides again the right type of generalization with respect to the existing framework [23] using Poisson structures.

As we will discuss in the next three examples, the port-Hamiltonian formulation of distributed-parameter systems is closely related to the general framework for describing basic distributed-parameter systems as systems of conservation laws, see e.g. [11], [37].

Example 4.1 (Inviscid Burger's equation). The viscous Burger's equation is a scalar parabolic equation defined on a one-dimensional spatial domain (interval) $Z=$ $[a, b] \subset \mathbb{R}$, with the state variable $\alpha(t, z) \in \mathbb{R}, z \in Z, t \in I$, where $I$ is an interval of $\mathbb{R}$, satisfying the partial differential equation

$$
\frac{\partial \alpha}{\partial t}+\alpha \frac{\partial \alpha}{\partial z}-v \frac{\partial^{2} \alpha}{\partial z^{2}}=0
$$

The inviscid $(v=0)$ Burger's equations may be alternatively expressed as

$$
\frac{\partial \alpha}{\partial t}+\frac{\partial}{\partial z} \beta=0
$$

where the state variable $\alpha(t, z)$ is called the conserved quantity and the function $\beta:=\frac{\alpha^{2}}{2}$ the flux variable. Eq. (46) is called a conservation law, since by integration one obtains the balance equation

$$
\frac{d}{d t} \int_{a}^{b} \alpha d z=\beta(a)-\beta(b) .
$$

Furthermore, according to the framework of Irreversible Thermodynamics [27], one may express the flux $\beta$ as a function of the generating force which is the variational derivative of some functional $H(\alpha)$ of the state variable. The variational derivative $\frac{\delta H}{\delta \alpha}$ of a functional $H(\alpha)$ is uniquely defined by the requirement

$$
H(\alpha+\varepsilon \eta)=H(\alpha)+\varepsilon \int_{a}^{b} \frac{\delta H}{\delta \alpha} \eta d z+O\left(\varepsilon^{2}\right)
$$

for any $\varepsilon \in \mathbb{R}$ and any smooth function $\eta(z, t)$ such that $\alpha+\varepsilon \eta$ satisfies the same boundary conditions as $\alpha$ [23]. For the inviscid Burger's equation one has $\beta=\frac{\delta H}{\delta \alpha}$, where

$$
H(\alpha)=\int_{a}^{b} \frac{\alpha^{3}}{6} d z
$$

Hence the inviscid Burger's equation may be also expressed as

$$
\frac{\partial \alpha}{\partial t}=-\frac{\partial}{\partial z} \frac{\delta H}{\delta \alpha} .
$$

This defines an infinite-dimensional Hamiltonian system in the sense of [23] with respect to the skew-symmetric operator $\frac{\partial}{\partial z}$ that is defined on the functions with support contained in the interior of the interval $Z$. 
From this formulation one derives that the Hamiltonian $H(\alpha)$ is another conserved quantity. Indeed, by integration by parts

$$
\frac{d}{d t} H=\int_{a}^{b} \frac{\delta H}{\delta \alpha} \cdot-\frac{\partial}{\partial z} \frac{\delta H}{\delta \alpha} d z=\frac{1}{2}\left(\beta^{2}(a)-\beta^{2}(b)\right) .
$$

We note that the right-hand side is a function of the flux variables evaluated at the boundary of the spatial domain $Z$.

The second example consists of a system of two conservations laws, corresponding to the case of two physical domains in interaction.

Example 4.2 (The p-system, cf. [11], [37]). The p-system is a model for e.g. a one-dimensional gas dynamics. Again, the spatial variable $z$ belongs to an interval $Z \subset \mathbb{R}$, while the dependent variables are the specific volume $v(t, z) \in \mathbb{R}^{+}$, the velocity $u(t, z)$ and the pressure functional $p(v)$ (which for instance in the case of an ideal gas with constant entropy is given by $p(v)=A v^{-\gamma}$ where $\gamma \geq 1$ ). The $p$-system is then defined by the following system of partial differential equations

$$
\begin{aligned}
\frac{\partial v}{\partial t}-\frac{\partial u}{\partial z} & =0 \\
\frac{\partial u}{\partial t}+\frac{\partial p(v)}{\partial z} & =0
\end{aligned}
$$

representing respectively conservation of mass and of momentum. By defining the state vector as $\alpha(t, z)=(v, u)^{T}$, and the vector-valued flux $\beta(t, z)=(-u, p(v))^{T}$ the p-system is rewritten as

$$
\frac{\partial \alpha}{\partial t}+\frac{\partial}{\partial z} \beta=0
$$

Again, according to the framework of Irreversible Thermodynamics, the flux vector may be written as function of the variational derivatives of some functional. Indeed, consider the energy functional $H(\alpha)=\int_{a}^{b} \mathscr{H}(v, u) d z$ where the energy density $\mathscr{H}(v, u)$ is given as the sum of the internal energy and the kinetic energy densities

$$
\mathscr{H}(v, u)=U(v)+\frac{u^{2}}{2}
$$

with $-\mathcal{U}(v)$ a primitive function of the pressure. (Note that for simplicity the mass density has been set equal to 1 , and hence no difference is made between the velocity and the momentum.) The flux vector $\beta$ may be expressed in terms of the variational derivatives of $H$ as

$$
\beta=\left(\begin{array}{cc}
0 & -1 \\
-1 & 0
\end{array}\right)\left(\begin{array}{l}
\frac{\delta H}{\delta v} \\
\frac{\delta H}{\delta u}
\end{array}\right) .
$$

The anti-diagonal matrix represents the canonical coupling between two physical domains: the kinetic and the potential (internal) domain. Thus the variational derivative 
of the total energy with respect to the state variable of one domain generates the flux variable for the other domain. Combining eqns. (53) and (55), the p-system may thus be written as the Hamiltonian system

$$
\left(\begin{array}{c}
\frac{\partial \alpha_{1}}{\partial t} \\
\frac{\partial \alpha_{2}}{\partial t}
\end{array}\right)=\left(\begin{array}{cc}
0 & -\frac{\partial}{\partial z} \\
-\frac{\partial}{\partial z} & 0
\end{array}\right)\left(\begin{array}{l}
\frac{\delta H}{\delta \alpha_{1}} \\
\frac{\delta H}{\delta \alpha_{2}}
\end{array}\right) .
$$

Using again integration by parts, one may derive the following energy balance equation:

$$
\frac{d}{d t} H=\beta_{1}(a) \beta_{2}(a)-\beta_{1}(b) \beta_{2}(b) .
$$

Notice again that the right-hand side of this power-balance equation is a quadratic function of the fluxes at the boundary of the spatial domain.

The last example is the vibrating string. It is again a system of two conservation laws representing the canonical interdomain coupling between the kinetic energy and the elastic potential energy. However in this example the classical choice of the state variables leads to express the total energy as a function of some of the spatial derivatives of the state variables.

Example 4.3 (Vibrating string). Consider an elastic string subject to traction forces at its ends, with spatial variable $z \in Z=[a, b] \subset \mathbb{R}$. Denote by $u(t, z)$ the displacement of the string and the velocity by $v(t, z)=\frac{\partial u}{\partial t}$. Using the vector of state variables $x(t, z)=(u, v)^{T}$, the dynamics of the vibrating string is described by the system of partial differential equations

$$
\frac{\partial x}{\partial t}=\left(\begin{array}{c}
v \\
\frac{1}{\mu} \frac{\partial}{\partial z}\left(T \frac{\partial u}{\partial z}\right)
\end{array}\right)
$$

where the first equation is simply the definition of the velocity and the second one is Newton's second law. Here $T$ denotes the elasticity modulus, and $\mu$ the mass density. The total energy is $H(x)=U(u)+K(v)$, where the elastic potential energy $U$ is a function of the strain $\frac{\partial u}{\partial z}(t, z)$

$$
U(u)=\int_{a}^{b} \frac{1}{2} T\left(\frac{\partial u}{\partial z}\right)^{2} d z
$$

and the kinetic energy $K$ depends on the velocity $v(t, z)=\frac{\partial u}{\partial t}$ as

$$
K(v)=\int_{a}^{b} \frac{1}{2} \mu v(t, z)^{2} d z .
$$

Thus the total system (58) may be expressed as

$$
\frac{\partial x}{\partial t}=\left(\begin{array}{cc}
0 & \frac{1}{\mu} \\
-\frac{1}{\mu} & 0
\end{array}\right)\left(\begin{array}{l}
\frac{\delta H}{\delta u} \\
\frac{\delta H}{\delta v}
\end{array}\right)
$$


where $\frac{\delta H}{\delta u}=\frac{\delta U}{\delta u}=-\frac{\partial}{\partial z}\left(T \frac{\partial u}{\partial z}\right)$ is the elastic force and $\frac{\delta H}{\delta v}=\frac{\delta K}{\delta v}=\mu v$ is the momentum.

In this formulation, the system is not anymore expressed as a system of conservation laws since the time-derivative of the state variables is a function of the variational derivatives of the energy directly, and not the spatial derivative of a function of the variational derivatives as before. Instead of being a simplification, this reveals a drawback for the case of non-zero energy flow through the boundary of the spatial domain. Indeed, in this case the variational derivative has to be completed by a boundary term since the Hamiltonian functional depends on the spatial derivatives of the state. For example, in the computation of the variational derivative of the elastic potential energy $U$ one obtains by integration by parts that $U(u+\varepsilon \eta)-U(u)$ equals

$$
-\varepsilon \int_{a}^{b} \frac{\partial}{\partial z}\left(T \frac{\partial u}{\partial z}\right) \eta d z+\varepsilon\left[\eta\left(T \frac{\partial u}{\partial z}\right)\right]_{a}^{b}+O\left(\varepsilon^{2}\right)
$$

and the second term in this expression constitutes an extra boundary term.

Alternatively we now formulate the vibrating string as a system of two conservation laws. Take as alternative vector of state variables $\alpha(t, z)=(\varepsilon, p)^{T}$, where $\varepsilon$ denotes the strain $\alpha_{1}=\varepsilon=\frac{\partial u}{\partial z}$ and $p$ denotes the momentum $\alpha_{2}=p=\mu v$. Recall that in these variables the total energy is written as

$$
H_{0}=\int_{a}^{b} \frac{1}{2}\left(T \alpha_{1}^{2}+\frac{1}{\mu} \alpha_{2}^{2}\right) d z
$$

and directly depends on the state variables and not on their spatial derivatives. Furthermore, one defines the flux variables to be the stress $\beta_{1}=\frac{\delta H_{0}}{\delta \alpha_{1}}=T \alpha_{1}$ and the velocity $\beta_{2}=\frac{\delta H_{0}}{\delta \alpha_{1}}=\frac{\alpha_{2}}{\mu}$. In matrix notation, the flux vector $\beta$ is thus expressed as a function of the variational derivatives $\frac{\delta H_{0}}{\delta \alpha}$ by

$$
\beta=\left(\begin{array}{cc}
0 & -1 \\
-1 & 0
\end{array}\right) \frac{\delta H_{0}}{\delta \alpha}
$$

Hence the vibrating string may be alternatively expressed by the system of two conservation laws

$$
\frac{\partial \alpha}{\partial t}=\left(\begin{array}{cc}
0 & \frac{\partial}{\partial z} \\
\frac{\partial}{\partial z} & 0
\end{array}\right) \frac{\delta H_{0}}{\delta \alpha}
$$

satisfying the power balance equation (57).

4.1. Systems of two conservation laws in interaction. Let us now consider the general class of distributed-parameter systems consisting of two conservation laws with the canonical coupling as in the above examples of the p-system and the vibrating string. Let the spatial domain $Z \subset \mathbb{R}^{n}$ be an $n$-dimensional smooth manifold with smooth $(n-1)$ )-dimensional boundary $\partial Z$. Denote by $\Omega^{k}(Z)$ the vector space of (differential) $k$-forms on $Z$ (respectively by $\Omega^{k}(\partial Z)$ the vector space of $k$-forms on $\partial Z$ ). 
Denote furthermore by $\Omega=\bigoplus_{k \geq 0} \Omega^{k}(Z)$ the algebra of differential forms over $Z$ and recall that it is endowed with an exterior product $\wedge$ and an exterior derivation $d$.

Definition 4.4. A system of conservation laws is defined by a set of conserved quantities $\alpha_{i} \in \Omega^{k_{i}}(Z), i \in\{1, \ldots, N\}$ where $N \in \mathbb{N}, k_{i} \in \mathbb{N}$, defining the state space $\mathcal{X}=\bigotimes_{i=1, \ldots, N} \Omega^{k_{i}}(Z)$, and satisfying a set of conservation laws

$$
\frac{\partial \alpha_{i}}{\partial t}+\mathrm{d} \beta_{i}=g_{i}
$$

where $\beta_{i} \in \Omega^{k_{i}-1}(Z)$ denote the set of fluxes and $g_{i} \in \Omega^{k_{i}}(Z)$ denote the set of distributed interaction forms. In general, the fluxes $\beta_{i}$ are defined by so-called closure equations

$$
\beta_{i}=J\left(\alpha_{i}, z\right), \quad i=1, \ldots, N
$$

leading to a closed form for the dynamics of the conserved quantities $\alpha_{i}$. The integral form of the conservation laws yields the following balance equations

$$
\frac{d}{d t} \int_{S} \alpha_{i}+\int_{\partial S} \beta_{i}=\int_{S} g_{i}
$$

for any surface $S \subset Z$ of dimension equal to the degree of $\alpha_{i}$.

Remark 4.5. A common case is that $Z=\mathbb{R}^{3}$ and that the conserved quantities are 3 -forms, that is, the balance equation is evaluated on volumes of the 3-dimensional space. In this case () takes in vector calculus notation the familiar form

$$
\frac{\partial \alpha_{i}}{\partial t}(z, t)+\operatorname{div}_{z} \beta_{i}=g_{i}, \quad i=1, \ldots, n .
$$

However, systems of conservation laws may correspond to differential forms of any degree. Maxwell's equations are an example where the conserved quantities are differential forms of degree 2 .

In the sequel, as in our examples sofar, we consider a particular class of systems of conservation laws where the closure equations are such that fluxes are linear functions of the variational derivatives of the Hamiltonian functional. First recall the general definition of the variational derivative of a functional $H(\alpha)$ with respect to the differential form $\alpha \in \Omega^{p}(Z)$ (generalizing the definition given before).

Definition 4.6. Consider a density function $\mathscr{H}: \Omega^{p}(Z) \times Z \rightarrow \Omega^{n}(Z)$ where $p \in$ $\{1, \ldots, n\}$, and denote by $H:=\int_{Z} \mathscr{H} \in \mathbb{R}$ the associated functional. Then the uniquely defined differential form $\frac{\delta H}{\delta \alpha} \in \Omega^{n-p}(Z)$ which satisfies for all $\Delta \alpha \in \Omega^{p}(Z)$ and $\varepsilon \in \mathbb{R}$

$$
H(\alpha+\varepsilon \Delta \alpha)=\int_{Z} \mathcal{H}(\alpha)+\varepsilon \int_{Z}\left[\frac{\delta H}{\delta \alpha} \wedge \Delta \alpha\right]+\mathrm{O}\left(\varepsilon^{2}\right)
$$

is called the variational derivative of $H$ with respect to $\alpha \in \Omega^{p}(Z)$. 
Definition 4.7. Systems of two conservation laws with canonical interdomain coupling are systems involving a pair of conserved quantities $\alpha_{p} \in \Omega^{p}(Z)$ and $\alpha_{q} \in$ $\Omega^{q}(Z)$, differential forms on the $n$-dimensional spatial domain $Z$ of degree $p$ and $q$ respectively, satisfying $p+q=n+1$ ('complementarity of degrees'). The closure equations generated by a Hamiltonian density function $\mathcal{H}: \Omega^{p}(Z) \times \Omega^{q}(Z) \times Z \rightarrow \Omega^{n}(Z)$ resulting in the Hamiltonian $H:=\int_{Z} \mathscr{H} \in \mathbb{R}$ are given by

$$
\left(\begin{array}{l}
\beta_{p} \\
\beta_{q}
\end{array}\right)=\varepsilon\left(\begin{array}{cc}
0 & (-1)^{r} \\
1 & 0
\end{array}\right)\left(\begin{array}{l}
\frac{\delta H}{\delta \alpha_{p}} \\
\frac{\delta H}{\delta \alpha_{q}}
\end{array}\right)
$$

where $r=p q+1, \varepsilon \in\{-1,+1\}$, depending on the sign convention of the considered physical domain.

Define the vector of flow variables to be the time-variation of the state, and the effort variables to be the variational derivatives

$$
\left(\begin{array}{c}
f_{p} \\
f_{q}
\end{array}\right)=\left(\begin{array}{c}
\frac{\partial \alpha_{p}}{\partial t} \\
\frac{\partial \alpha_{q}}{\partial t}
\end{array}\right), \quad\left(\begin{array}{c}
e_{p} \\
e_{q}
\end{array}\right)=\left(\begin{array}{c}
\frac{\delta H}{\delta \alpha_{p}} \\
\frac{\delta H}{\delta \alpha_{q}}
\end{array}\right) .
$$

Their product equals again the time-variation of the Hamiltonian

$$
\frac{d H}{d t}=\int_{Z}\left(e_{p} \wedge f_{p}+e_{q} \wedge f_{q}\right) .
$$

Using the conservation laws (4.5) for $g_{i}=0$, the closure relations (70) and the properties of the exterior derivative $d$ and Stokes' theorem, one obtains

$$
\begin{aligned}
\frac{d H}{d t} & =\int_{Z} \varepsilon \beta_{q} \wedge\left(-d \beta_{p}\right)+(-1)^{r} \beta_{p} \wedge \varepsilon\left(-d \beta_{q}\right) \\
& =-\varepsilon \int_{Z} \beta_{q} \wedge d \beta_{p}+(-1)^{q} \beta_{q} \wedge d \beta_{p}=-\varepsilon \int_{\partial Z} \beta_{q} \wedge \beta_{p} .
\end{aligned}
$$

Finally, as before we define the power-conjugated pair of flow and effort variables on the boundary as the restriction of the flux variables to the boundary $\partial Z$ of the domain $Z$ :

$$
\left(\begin{array}{c}
f_{\partial} \\
e_{\partial}
\end{array}\right)=\left(\begin{array}{c}
\left.\beta_{q}\right|_{\partial Z} \\
\left.\beta_{p}\right|_{\partial Z}
\end{array}\right)
$$

On the total space of power-conjugated variables, that is, the differential forms $\left(f_{p}, e_{p}\right)$ and $\left(f_{q}, e_{q}\right)$ on the domain $Z$ and the differential forms $\left(f_{\partial}, e_{\partial}\right)$ defined on the boundary $\partial Z$, one defines an interconnection structure by Eqn. (74) together with

$$
\left(\begin{array}{l}
f_{q} \\
f_{p}
\end{array}\right)=\varepsilon\left(\begin{array}{cc}
0 & (-1)^{r} d \\
d & 0
\end{array}\right)\left(\begin{array}{l}
e_{q} \\
e_{p}
\end{array}\right) .
$$

This interconnection can be formalized as a special type of Dirac structure, called Stokes-Dirac structure, leading to the definition of distributed-parameter port-Hamiltonian systems [35]. 


\section{Concluding remarks}

We have surveyed some of the recently developed theory of port-Hamiltonian systems; for further applications towards modeling, analysis, simulation and control we refer to the literature cited below.

From the geometric point of view many questions regarding port-Hamiltonian systems are waiting to be investigated. A theory of symmetry and reduction of portHamiltonian systems has been explored in [29], [1], while some questions concerning integrability of Dirac structures have been studied in [7]. A main question for distributed-parameter port-Hamiltonian systems concerns the relation with variational calculus.

\section{References}

[1] Blankenstein, G., van der Schaft, A. J., Symmetry and reduction in implicit generalized Hamiltonian systems. Rep. Math. Phys. 47 (2001), 57-100.

[2] Bloch, A. M. and Crouch, P. E., Representations of Dirac structures on vector spaces and nonlinear $L C$ circuits. In Differential geometry and control (ed. by G. Ferreyra, R. Gardner, H. Hermes, H. Sussmann), Proc. Sympos. Pure Math. 64, Amer. Math. Soc., Providence, RI, 1999, 103-117.

[3] Breedveld, P. C., Physical systems theory in terms of bond graphs. PhD thesis, University of Twente, Faculty of Electrical Engineering, 1984.

[4] R. W. Brockett, Control theory and analytical mechanics. In Geometric Control Theory (ed. by C. Martin, R. Hermann), Lie Groups: History, Frontiers and Applications VII, Math. Sci. Press, Brookline 1977, 1-46.

[5] Cervera, J., van der Schaft, A. J., Banos, A., Interconnection of port-Hamiltonian systems and composition of Dirac structures. Automatica, submitted.

[6] Courant, T. J., Dirac manifolds. Trans. Amer. Math. Soc. 319 (1990), 631-661.

[7] Dalsmo, M., and van der Schaft, A. J., On representations and integrability of mathematical structures in energy-conserving physical systems. SIAM J. Control Optim. 37 (1999), 54-91.

[8] Dorfman, I., Dirac Structures and Integrability of Nonlinear Evolution Equations. John Wiley, Chichester 1993.

[9] Duindam, V., Blankenstein, G., Stramigioli, S., Port-Based Modeling and Analysis of Snakeboard Locomotion. In Proceedings 16th International Symposium on Mathematical Theory of Networks and Systems (MTNS2004), Leuven 2004.

[10] Escobar, G., van der Schaft, A. J., and Ortega, R., A Hamiltonian viewpoint in the modelling of switching power converters. Automatica 35 (Special Issue on Hybrid Systems) (1999), $445-452$.

[11] Godlewsky, E., and Raviart, P., Numerical Approximation of Hyperbolic Systems of Conservation Laws. Appl. Math. Sci. 118, Springer-Verlag, New York 1996.

[12] Golo, G., van der Schaft, A. J., Breedveld, P. C., Maschke, B. M., Hamiltonian formulation of bond graphs. In Nonlinear and Hybrid Systems in Automotive Control (ed. by R. Johansson, A. Rantzer), Springer-Verlag, London 2003, 351-372. 
[13] Hogan, N., Impedance Control: An approach to manipulation. J. Dyn. Systems, Measurements Control 107 (1985), 1-24.

[14] Karnopp, D. C., Margolis, D. L., and Rosenberg, R. C., System Dynamics, A Unified Approach. John Wiley and Sons, 1990.

[15] Kugi, A., Kemmetmüller, W., Impedance control of Hydraulic piston actuators. In Proceedings 6th IFAC Symposium on Nonlinear Control Systems (NOLCOS 2004), 1-3 September, Stuttgart.

[16] Marsden, J. E., and Ratiu, T. S., Introduction to Mechanics and Symmetry. Texts Appl. Math. 17, Springer-Verlag, New York 1994.

[17] Maschke, B. M., and van der Schaft, A. J., Port-controlled Hamiltonian systems: Modelling origins and system-theoretic properties. In Proc. 2nd IFAC NOLCOS, Bordeaux 1992, 282-288.

[18] Maschke, B. M., and van der Schaft, A. J., System-theoretic properties of port-controlled Hamiltonian systems. In Systems and Networks: Mathematical Theory and Applications, Vol. II, Akademie-Verlag, Berlin 1994, 349-352.

[19] Maschke, B. M., van der Schaft, A. J., and Breedveld, P. C., An intrinsic Hamiltonian formulation of network dynamics: non-standard Poisson structures and gyrators. J. Franklin Inst. 329 (1992), 923-966.

[20] Maschke, B. M., van der Schaft, A. J., and Breedveld, P. C., An intrinsic Hamiltonian formulation of the dynamics of LC-circuits. IEEE Trans. Circuits Systems I Fund. Theory Appl. 42 (1995), 73-82.

[21] Maschke, B. M., Ortega, R., and van der Schaft, A. J., Energy-based Lyapunov functions for forced Hamiltonian systems with dissipation. IEEE Trans. Automat. Control 45 (2000), 1498-1502.

[22] Neimark, J. I., and Fufaev, N. A., Dynamics of Nonholonomic Systems. Transl. Math. Monogr. 33, Amer. Math. Soc., Providence, RI, 1972.

[23] Olver, P. J., Applications of Lie Groups to Differential Equations. Second edition, Grad. Texts in Math. 107, Springer-Verlag, New York 1993.

[24] Ortega, R., van der Schaft, A. J., Maschke, B. M., and Escobar, G., Interconnection and damping assignment passivity-based control of port-controlled Hamiltonian systems. $\mathrm{Au}$ tomatica 38 (2002), 585-596.

[25] Ortega, R., van der Schaft, A. J., Mareels, I., and Maschke, B. M., Putting energy back in control. IEEE Control Syst. Mag. 21 (2001), 18-33.

[26] Paynter, H. M., Analysis and design of engineering systems. M.I.T. Press, Cambridge, MA, 1960.

[27] Prigogine, I., Introduction to Thermodynamics of Irreversible Processes. John Wiley and Sons, 1962.

[28] van der Schaft, A. J., System theoretic properties of physical systems. CWI Tract 3, Centre for Mathematics and Informatics, Amsterdam 1984.

[29] van der Schaft, A. J., Implicit Hamiltonian systems with symmetry. Rep. Math. Phys. 41 (1998), 203-221.

[30] van der Schaft, A. J., Interconnection and geometry. In The Mathematics of Systems and Control, From Intelligent Control to Behavioral Systems (ed. by J. W. Polderman, H. L. Trentelman), Groningen 1999, 203-218. 
[31] van der Schaft, A. J., L $L_{2}$-Gain and Passivity Techniques in Nonlinear Control. 2nd revised and enlarged edition, Comm. Control Engrg. Ser., Springer-Verlag, London 2000 (first edition; Lecture Notes in Control and Inform. Sci. 218, Springer-Verlag, London 1996).

[32] van der Schaft, A. J., and Maschke, B. M., On the Hamiltonian formulation of nonholonomic mechanical systems. Rep. Math. Phys. 34 (1994), 225-233.

[33] van der Schaft, A. J., and Maschke, B. M., The Hamiltonian formulation of energy conserving physical systems with external ports. AE ̈U-Arch. Elektron. Übertragungstech. 49 (1995), 362-371.

[34] van der Schaft, A. J., and Maschke, B. M., Interconnected Mechanica5a Systems, Part I: Geometry of Interconnection and implicit Hamiltonian Systems. In Modelling and Control of Mechanical Systems (ed. by A. Astolfi, D. J. N. Limebeer, C. Melchiorri, A. Tornambe, R. B. Vinter), Imperial College Press, London 1997, 1-15.

[35] van der Schaft, A. J., Maschke, B. M., Hamiltonian representation of distributed parameter systems with boundary energy flow. J. Geom. Phys. 42 (2002), 166-194.

[36] Stramigioli, S., Modeling and IPC control of Interactive Mechanical Systems: a coordinate free approach. Lecture Notes in Control and Inform. Sci. 266, Springer-Verlag, London 2001.

[37] Serre, D., Systems of Conservation Laws. Cambridge University Press, Cambridge 1999.

[38] Willems, J. C., Dissipative dynamical systems - Part I: General Theory. Arch. Rational Mech. Anal. 45 (1972), 321-351.

Department of Mathematics and Computing Science, University of Groningen, P.O. Box 800, 9700 AV Groningen, The Netherlands

E-mail: A.J.van.der.Schaft@math.rug.nl 